\title{
Le Chasse-ennuy de Louis Garon : pour une poétique du rire
}

\section{Stéphane Macé}

\section{OpenEdition}

12 Journals

\section{Édition électronique}

URL : http://journals.openedition.org/recherchestravaux/255

DOI : 10.4000/recherchestravaux.255

ISSN : 1969-6434

\section{Éditeur}

UGA Éditions/Université Grenoble Alpes

\section{Édition imprimée}

Date de publication : 30 mai 2005

Pagination : 13-24

ISBN : 0151-1874

ISSN : 0151-1874

\section{Référence électronique}

Stéphane Macé, "Le Chasse-ennuy de Louis Garon : pour une poétique du rire ", Recherches \& Travaux [En ligne], 67 | 2005, mis en ligne le 30 septembre 2008, consulté le 08 septembre 2020. URL : http:// journals.openedition.org/recherchestravaux/255; DOI : https://doi.org/10.4000/recherchestravaux. 255 
Stéphane MACÉ

Université Stendhal - Grenoble 3

\section{Le Chasse-ennuy de Louis Garon: pour une poétique du rire}

Dans la version actualisée du Dictionnaire des Lettres françaises ${ }^{\mathrm{I}}$, on peut trouver, sous la plume de Jean Serroy, une brève notice consacrée à Louis Garon (I574-I63I) : cet écrivain lyonnais, d'origine genevoise, est l'auteur d'un copieux volume d'anecdotes intitulé Le Chasse-ennuy ${ }^{2}$, qu'il fallait assurément toute la curiosité de notre collègue grenoblois pour chasser de notre oubli! L'ouvrage est organisé en cinq "centuries », c'est-à-dire en cinq groupes de cent petits récits généralement classés par affinités thématiques, en fonction notamment des corps professionnels des personnages (rois et grands seigneurs, hommes de loi, médecins, hommes d'Église, étudiants ou paysans), mais aussi, à l'occasion, selon leur nationalité. Cette œuvre hybride emprunte à différentes traditions, qu’il s'agisse de la littérature facétieuse, de l'anecdote, ou de la littérature morale. Nous avons donc affaire à un texte assez étrange, dont il nous faudra expliquer le titre, sans doute moins anodin qu'il y paraît. Un dispositif très complet de «seuils» (dédicace, avis au lecteur, avant-propos, poèmes d'hommage) s'efforce d'en rendre compte, et tend à orienter notre lecture à la lumière d'une véritable poétique du rire, construite pas à pas, éventuellement par à-coups ou par retouches successives. C’est ce

I. Dictionnaire des Lettres françaises, Le XVII siècle, sous la dir. de P. Dandrey, Paris, Fayard / Librairie générale française (La Pochothèque), 1996 (révision de la version de 1951 préparée par A. Pauphilet, L. Pichard et R. Barroux, Paris, Fayard, I95I).

2. L. Garon, Le Chasse-ennuy, ou l'entretien des honnestes compagnies, Paris, Bontemps, 1600. L'édition originale porte l'étrange mention «trop-tost», qui signale que la date de parution est vraisemblablement fausse: certaines anecdotes semblent en effet appartenir à une époque légèrement plus tardive; par exemple, le $\$$ LXXXV de la première "centurie» retrace une anecdote datée précisément de "L'an I608. Le is de Iuillet sur le Midy» (p. 85-86). Le texte sera réédité en I633, I645 (Paris, C. Grisot) et I652 (Rouen, J. Cailloué). L'indication d'une date erronée est sans doute volontaire, car l'insertion de "fausses pistes", de plaisanteries ou de clins d'œil ironiques correspond à une pratique courante chez les auteurs de littérature facétieuse. 
cheminement complexe que nous voudrions tenter de reconstituer, avant d'isoler, dans la pratique du texte même, quelques caractéristiques marquantes de celle de l'auteur.

Le premier «seuil» du texte (si l'on excepte le titre lui-même) est une dédicace liminaire à un personnage considérable: "Maistre Jean Durand, Conseiller du Roy, Maistre et Correcteur en la Chambre des Comptes de Dauphiné, \& Secretaire de Monseigneur d'Halincourt, Cheualier des Ordres du Roy, Gouuerneur, \& Lieutenant General pour sa Majesté en la Ville de Lyon, pays de Lyonnois, Forests, \& Beaujollois. " La mention scrupuleuse de ces nombreux titres va sans doute au-delà de la déférence de convention: le rappel des activités du dédicataire justifie aussi par avance et de façon indirecte la part importante des anecdotes empruntées au petit monde des hommes de loi, ou la forte représentation des habitants de Lyon ou du Dauphiné.

Surtout, ce texte liminaire se veut une première explicitation du titre; celui-ci, on l'aura noté, s'apparente à un néologisme formé sur le modèle lexical d'un mot composé. Le sens, bien sûr, n'en est pas fort mystérieux, mais les «seuils» du recueil ont précisément pour fonction de lui donner davantage de sens ou d' "épaisseur»:

Ce ne sont pas des ennuis que ie vous presente; vostre humeur toute sincere ne les pourroit supporter, ny treuuer de bon goust; Ce sont plustost des Verueines consacrées à la ioye, dont vous estes le Dieu tutelaire: ou des Angeliques odoriferantes d'allegresses, dont vous remplissez les cœurs de ceux qui ont le bonheur de vous aborder: Mais ie diray mieux; c'est vn Lotus, un Chasse ennuy, qui vous pourra donner quelque diuertissement, ou plustost quelque agreable recreatiō aux heures perduës de vos serieuses occupations (si tât est que vous en puißiez auoir de perduës parmy tant d'emplois qui tiennent vostre esprit attaché aux affaires qui vous sont commises:) Vous n'y pourrez rien perdre, comme ie croy en le lisant \& relisant, puis que vous y verrez des poincts d'Estat fort remarquables, tels que vous maniez, \& des réponses subtiles \& aiguës des plus Grands de tous les temps; mais sur tout du nostre, entremeslez de diuerses galantises fort recreatiues, qui pourront, comme ie me promets, donner de la ioye $\&$ du contentement aux plus melancoliques, \& leur seruir d'Hellebore pour déchasser les mornes humeurs de la tristesse. $[\ldots]^{3}$

Deux traits, dans cette page, semblent particulièrement remarquables: on note d'emblée le traitement métaphorique extrêmement dense, qui assimile l'ouvrage à un certain nombre de plantes médicinales, soit successivement la verveine, l'angélique, le lotus, et enfin l'hellébore. Il semble que l'ordre de présentation ne soit pas purement aléatoire: si l'on confronte les définitions

3. Non paginé. 
proposées par les trois grands dictionnaires de la fin du siècle 4 , on constate que Garon mentionne les quatre plantes par ordre croissant d'efficacité. Plus exactement, les trois premières ne retiennent guère l'attention des lexicographes, alors que les vertus curatives de l'hellébore font l'objet de développements beaucoup plus conséquents. Pour la verveine, on se contente de rappeler l'usage qu'en pouvaient faire les Anciens dans leurs sacrifices païens - avec, semble-t-il, quelque prise de distance vis-à-vis de ces croyances périmées'. L'angélique est simplement qualifiée d' "odoriférante» par Richelet, qui se satisfait d'une description physique de la plante, et renvoie aux ouvrages spécialisés pour de plus amples informations ${ }^{6}$. Le Dictionnaire de l'Académie expédie le sens botanique en une ligne: "Est aussi une sorte de racine fort chaude.» Sur le lotus, rien chez Richelet, non plus que sous la plume des Immortels. Furetière signale simplement qu'il s'agit d'une plante médicinale, mais examine simplement les caractéristiques du végétal sans s'attarder sur ses propriétés réelles ou supposées. Tout change dès que l'on passe à l'hellébore: cette plante est auréolée d'un prestige que les trois autres ne sauraient lui disputer. Si Richelet, une fois de plus, renvoie simplement à la littérature spécialisée, Furetière inverse la perspective, évoquant les vertus médicinales de la plante avant même de procéder à sa description technique:

Plante medicinale qui sert à purger, \& sur tout les humeurs melancoliques $\&$ les vapeurs qui offusquent le cerveau. [...]

On dit proverbialement, qu'un homme a besoin d'hellebore, pour luy reprocher qu'il a quelque grain de folie.

Ses confrères et rivaux de l'Académie lui emboîtent le pas, proposant une définition assez proche:

Herbe medicinale, que l'on croit propre à guerir la folie. Ellebore blanc, ellebore noir. On dit prov. qu'Un homme a besoin d'ellebore, pour dire, qu'il a l'esprit troublé, \& qu'il n'est pas en son bon sens.

Description assez proche, certes, mais pas totalement concordante; la mélancolie n'est tout de même pas la même chose que la folie. Nous aurons à

4. Richelet, I680; Furetière, I690; Dictionnaire de l'Académie, I694. L'anachronisme de cette triple référence n'est guère gênant, dans la mesure où, en matière de botanique, les lexicographes reproduisent bien souvent un savoir ou une doxa relativement stables.

5. Richelet: "Les Anciens Romains se servoient de la verveine dans les sacrifices, \& ils croioient qu'elle étoit capable de chasser les malins esprits de leurs maisons.»/Académie: «Sorte d'herbe dont les anciens se servoient dans leurs sacrifices, dans leurs ceremonies superstitieuses.»

6. Son ouvrage de référence, pour tout ce qui touche à la botanique, est le livre de Dalechamp sur les plantes. Selon Olivier de Serres, (Le Théatre de l'agriculture et des champs) le nom de l'angélique lui vient «des vertus qu'elle a contre les venims» (comprendre, les poisons en général). 
revenir sur ce point, qui constitue, par effet de balancier, l'un des fondements de la poétique du rire dont se réclame Louis Garon.

Pour le moment, attardons-nous sur la prolifération de ces herbes médicinales et sur la façon dont elles sont présentées. La métaphore filée - et c'est le second trait remarquable de cette dédicace - ne se construit pas de façon absolument linéaire et cohérente, mais sur le mode de la correction permanente, ce dont la syntaxe même de la phrase porte distinctement la trace. La dédicace s'ouvre sur une description négative, qui autorise un effet d'antithèse marqué («Ce ne sont pas» / «Ce sont»). Les conjonctions, utilisées en tête de proposition ou après une rupture rythmique forte, soulignent les mouvements de réorientation du propos ou les effets de surenchère, glissant ainsi vers un fonctionnement adverbial ("ou des Angeliques odoriferantes» / "Mais ie diray mieux»). La syntaxe de l'apposition ("un Lotus, un Chasse ennuy") accentue discrètement une fausse équivalence - ce qui est le principe même de la figure de métaphore. Les adverbes de modalisation, la subordonnée concessive, et plus largement les propositions incidentes, entendent relativiser le propos. C'est là une syntaxe assez surprenante, qui déploie aux yeux du lecteur de nombreux méandres. Il semble que ce choix ne soit pas dû au hasard, mais réponde à une véritable stratégie: il s'agit d'un texte apéritif, qui présente d'emblée la matière du livre sous le signe de la diversité - érigée, bien avant La Fontaine, en devise implicite.

Cette approche syntaxique permet sans doute d'aborder sous un autre angle la métaphore des plantes médicinales: il est probable que Garon mobilise ici une autre métaphore bien connue, liée à la topique générique de nombreux recueils de formes brèves relevant d'une esthétique du mélange - ou, plus généralement, de toute œuvre de nature composite. Il est fréquent, à l'aube de l'âge baroque mais aussi dès la Renaissance, de comparer de tels textes à des «fricassées", à des «ragoûts», ou autres «salades» qui proposent une variation sur un même paradigme alimentaire. C'est à cette tradition que pense visiblement Louis Garon, et le «seuil » suivant de l'ouvrage (l'adresse au lecteur) assure une compréhension rétrospective des deux dimensions de la métaphore:

En fin, mon cher Lecteur, ie vous presente vne Salade cõposée de plusieurs sortes d'herbes: il ne tient qu'à vous d'y apporter du sel $\&$ de l'huile pour les faire mieux venir à vostre goust. Ie ne doute point qu'il ne se trouue beaucoup d'esprits dégoutez, $\&$ difficiles à contenter, qui reietteront ce mien petit œuure, comme ne reuenant à leur palais: mais pourueu que i'aye l'approbation \& l'acclamation des gens de bien, ce m'est assez. A dieu, \& attendez vne seconde Partie, si vous receuez celle-cy de bon cœur.7

7. Non paginé. Il s'agit de la conclusion de l'avis au lecteur. 
Il faut bien sûr lire les deux textes en relation l'un avec l'autre. Le texte est une salade composée, mais une salade aromatique, composée d'herbes aux vertus cathartiques ou curatives. La métaphore, sous la plume de Garon, se développe ainsi selon deux axes distincts mais complémentaires.

À ce modèle de lecture quelque peu complexe, ajoutons encore une strate: dans l'avis au lecteur, l'auteur revient sur le titre de l'ouvrage, et, proposant une seconde justification, en attribue cette fois le choix à une expérience personnelle douloureuse:

AMY Lecteur, La Vertu ne peut demeurer oisive, ni croupir sous la feneantise d'vn ingrat repos: quoy qu'elle soit trauaillée, elle est tousiours viue $\&$ vigoureuse: les incommoditez de cette vie luy donnent de l'ennuy, mais parmy ses destresses elle ne laisse pas de se resiouïr, \& de se donner de l'allegresse au milieu de ses disgraces, pour faire voir qu'elle est autant maistresse de ses incommoditez comme elle est subjecte à beaucoup de trauerses. Ie puis dire de moy-mesme que durant la calamité d'vne furieuse maladie de goutes, ou enuiron, si mon corps a esté affligé, mô ame n'en est pas demeurée moins vigoureuse. Ie souffroy d'vn costé, \& me consoloy de l'autre; \& si le mal m’a porté quelques fois iusques aux derniers termes de la patience: i'ay tasché d'autre costé de contrebatre ces miseres par des moyens qui m'estoient entierement incogneus. Cet aiguillon m’a esté fort sensible mais aussi m'a il esté (comme ie croy) aucunement fauorable: car cerchãt à chasser mes Ennuis, i'ay dressé vn ChasseEnnuy, pour me seruir à me desennuyer: $\&$ croy-ie qu'il pourra seruir à beaucoup d'autres, qui pourront prendre de la consolation en mes consolations, $\&$ comme moy faire estat de se rire de tout ce que le monde nous peut representer de bon, ou de mauuais goust. Ie peus dốc dire, que durant ce temps-là ie me suis entierement plongé comme dans vn bain salutaire en la lecture de plusieurs bons Autheurs, où i'ay trouué beaucoup de remedes pour adoucir mes douleurs: mais entre tous ie n'ay rien trouué qui ait tant contenté mon humeur melancolique, ni regaillardy mes esprits, que les responses aigues \& promptes, sans aucune premeditation, de plusieurs rares esprits en des rencontres inopinées: mais sur tout de ceux de nostre temps, \& qui n'ont point encore esté veuës.

Cet épisode «autobiographique» - que cette histoire de la genèse du texte soit réelle ou supposée - est intéressant à plus d'un titre. Il permet, d'une part, de concevoir le texte comme un objet complexe, «à géométrie variable», pouvant viser plusieurs publics: il s'agit à l'origine d'un texte intime, destiné à son seul auteur, et présenté comme un "médicament littéraire» élaboré pour contrarier les ennuis de la goutte - maladie physique plutôt que morale, $a$ priori. Mais dès lors que le livre s'adresse à Maître Jean Durand, le rire thérapeutique devient pur "divertissement» (au sens étymologique du terme) et permet de congédier les tracas du quotidien : c'est en ce sens, probablement, que doit être comprise la métaphore du lotus, fleur de l'oubli depuis les Lotophages de l'Odyssée : 
[...] c'est vn Lotus, un Chasse ennuy, qui vous pourra donner quelque diuertissement, ou plustost quelque agreable recreatiô aux heures perduës de vos serieuses occupations.

Si remède il y a, il ne peut s'agir ici que d'un remède «littéraire», tirant précisément son efficace d'une réminiscence qui ne saurait être que littéraire. Enfin, destiné à un public plus large, le rire récupère ses vertus proprement médicinales, et devient un antidote privilégié contre la mélancolie.

Sur ce point, l'auteur tend à s'inscrire dans la même sphère que son propre public, par une étrange contamination entre la maladie de la goutte et la mélancolie, dont il nous dit souffrir également. Cette mise en relation pourrait sembler étrange au lecteur moderne, mais ne doit pourtant pas surprendre, à une époque où la mélancolie est appréhendée selon des critères très différents des nôtres: la goutte, nous dit Furetière, est une «Maladie causée par la fluxion d'une humeur acre sur les articles ou jointures du corps.» La mélancolie est elle aussi, comme on sait, une affaire d'humeurs. Le problème est trop complexe pour être traité dans l'espace d'un simple article, et la littérature critique, sur ce point, est particulièrement abondante ${ }^{8}$. Signalons simplement que les connaissances médicales de l'époque sont sur ce point très généralement partagées, et que les œuvres littéraires s'en font volontiers l'écho, même lorsque leur sujet est a priori éloigné de thèmes proprement scientifiques ou médicaux 9 .

Très souvent, la mélancolie est placée sous le signe de l'ambiguïté: passion destructrice, elle peut aussi devenir particulièrement féconde:

Les melãcholiques, écrit le Sieur de Mousé, sont tenus pour les plus capables de grandes charges \& hautes entreprises, car ils sont plus ingenieux que tous les autres. ${ }^{\mathrm{IO}}$

On conçoit que Garon ait tout intérêt à ce que l'image de l'esprit mélancolique éclipse celle de malade de la goutte! Mais voisiner avec le génie

8. On pense bien entendu à la magistrale somme de P. Dandrey, Molière et la médecine, Paris, Klincksieck, 1998 (2 vol.).

9. Voir par exemple Les Larmes de Floride essuyees par Minerve du Sieur de Mousé, Paris, L. Boulanger, 1927, qui consacre une vingtaine de pages à un exposé de la théorie des humeurs (p. 205-223). Citons le cœur de l'exposé consacré à la mélancolie:

"[...] il y a trois especes de melancholie: l'vne desquelles est du tout grossiere $\&$ terrestre froide $\&$ seiche. Lautre qui est chaude $\&$ aduste $\&$ appellée bile noire: $\&$ la derniere qui est meslée auec vn peu de sang; tenant toutesfois plus du sec que de l'humide.

La premiere rend les hommes du tout grossiers \& tardifs en leurs actions de corps \& desprit, timides, paresseux $\&$ sans entendement, La seconde les faict deuenir furieux, violents, turbulents $\&$ brouillons $\&$ incapables de toutes charges: mais la troisiesme est tres-louable $\&$ tres noble rendant les hommes ingenieux \& les faisant exceller sur tous les autres: d'autant que leur cerueau n'est ny trop mol ny trop dur quoy que la seicheresse y domine» (p. 210-2I2).

IO. Ibid., p. 2 IO. 
comporte quelques risques, et la mélancolie n'est pas non plus très éloignée de la folie. Et ce que nous dit Cotin du mélancolique amoureux pourrait être vrai du mélancolique en général:

Voila comme sa melancholie naturellement \& sans effort le met au dessus de tous les changemens de la fortune, $\&$ l'exempte des passions que les bons $\&$ mauuais succez apportent ordinairement avec eux. Extraordinaire sagesse qui semble en effect d'vne extraordinaire folie! nompareil accident, où l'on doit à sa maladie la principale cause de sa santé!"r

On comprend au vu de cette dangereuse proximité que l'hellébore puisse être considéré par les disciples d'Hippocrate comme un remède commun à la mélancolie et à la folie. En réponse à ce mal ambigu, à la fois fécond et destructeur, Louis Garon propose une thérapie par le rire. Encore faut-il en préciser la valeur.

Le rire est aussi quelque chose de foncièrement ambigu: il peut être un indice ou un révélateur de la diversité humaine, se démultipliant en fonction des individus en d'infinies variations ${ }^{12}$; il est le signe extérieur d'un état d'âme tout intérieur; il se situe à la frontière du verbal et du non verbal; il appartient en propre à l'homme, et peut à ce titre constituer une marque de sa supériorité par rapport aux autres animaux; mais dans le même temps, le rire peut devenir incontrôlable, et être perçu comme un signe de possession diabolique (le Christ est réputé ne pas avoir ri).

Garon, semble-t-il, est bien conscient de ces ambiguïtés. L'auteur prévoit, en fonction du goût ou des humeurs de chacun, de possibles variations d'intensité. Davantage, cette gradualité du rire est inscrite dans son propos même: l'auguste dédicataire du texte ne s'autorisera guère qu'une "agreable recreation", certes opposée au sérieux du quotidien, mais qui reste bien éloignée d'un rire à gorge déployée! Le mélancolique se permettra quant à lui des manifestations inversement proportionnelles à son mal, selon une gamme qui peut parcourir tous les degrés, de la simple «consolation" à la franche «allégresse».

Il faut aussi noter que l'avis au lecteur, de façon très spectaculaire, s'ouvre sur une quasi-allégorie de la Vertu. C'est pour l'auteur une façon de légitimer le rire: non plus rire banal, gratuit, et partant toujours suspect, mais rire autorisé,

II. Les Regrets d'Aristee, sur le Trespas de Daphnis, Paris, I62I, p. 58-59.

I2. "En l'espèce des hommes, il y a autant de visages différents, qu'il y a de figures au monde; autant de diversités tant au parler qu'à la voix, autant de divers ris.» (L. Joubert, Traité du Ris, Paris, I560, cité par D. Bertrand, «Rire et sémiotique corporelle de la Renaissance à l'Âge classique: une nouvelle lisibilité», dans La Peinture des Passions de la Renaissance à l'Âge classique, Actes du colloque de Saint-Étienne, IO-I2 avril I991, textes réunis par B. Yon, Publications de l'université de Saint-Étienne, 1995, p. 283. 
contrôlé, cathartique et utilitaire, en un mot, un rire moral. On relève d'ailleurs dans les seuils, comme dans le corps même du texte, d'assez nombreux emplois pronominaux du verbe: il s'agit de «se rire», et non plus de «rire». La distinction est d'importance: sans qu'il le précise autrement que par le choix des exemples en langue, Furetière semble réserver la forme pronominale du verbe à un usage moralement orienté. Entre la première entrée du verbe, consacrée aux manifestations physiologiques du rire, et la troisième, qui exploite le sème de divertissement, il insère un sens articulé à l'idée de raillerie (avec toute la complexité que ce terme peut revêtir au XVII siècle) :

Rire, signifie aussi, Se mocquer de quelqu'un, le railler, ou le mespriser. Dieu se rit des folles entreprises des hommes. [...] Un libertin se rit de toutes les remontrances, de toutes les menaces qu'on luy fait. [...]

Le rire a ici à voir avec une pratique sociale et devient, sous certaines de ses formes, typique de l'idéal de l'honnête homme, auquel l'ouvrage de Nicolas Faret donnera ses lettres de noblesse en I630. Il se fait l'expression de l'urbanité, définie par Balzac comme

[...] une adresse à toucher l'esprit par je ne sais quoi de piquant, mais dont la piqûre est agréable à celui qui la reçoit, parce qu'elle chatouille et n'entame pas, parce qu'elle laisse un aiguillon sans douleur et réveille la partie que la médisance blesse..$^{13}$

Il est à noter que les termes de "pointe», d' «aiguillon", que les qualificatifs de "piquant» ou d' «aigu» font fréquemment retour sous la plume de Garon, que ce soit dans les «seuils» du texte, au niveau des titres qui accompagnent les différentes anecdotes, ou dans ces petits récits eux-mêmes. Il n'est pas jusqu'aux poèmes d'hommage liminaires qui ne saluent ces qualités, révélatrices d'une certaine forme de modernité. Enfin, le terme de "galantise», qui apparaît dans la dédicace, se réfere à un idéal tout à la fois social et littéraire qui connaîtra bientôt un succès considérable. En cela, l'œuvre de Garon est un maillon assez intéressant entre l'héritage renaissant de la littérature facétieuse, auquel il doit encore beaucoup (et qui reste peut-être même l'horizon de lecture prioritaire), et une sensibilité émergente, celle des cercles mondains de la modernité.

Pour être légitime, le rire doit être justifié par une visée cathartique et morale, et Garon se signale par la recherche permanente d'une forme d'équilibre: un troisième «seuil» autographe, sous la forme d'un "avant-propos», recentre la réflexion autour de cette thématique unique - la dédicace et l'avis au lecteur affichaient à l'inverse un caractère mixte, en respectant ces fourches

13. Guez de Balzac, cité par R. Zuber, Les Émerveillements de la Raison, Paris, Klincksieck, 1997, p. 147. 
caudines des textes préfaciels que sont la justification du titre, le récit de la genèse de l'ouvrage, les déclarations d'intentions ou la sélection des publics ${ }^{14}$. Le propos est d'abord une légitimation générale du rire, sous un angle philosophique ou anthropologique - variation sur un air assez connu :

Les philosophes pour distinguer l'homme d'entre les animaux irraisonnables l'ont appellé, Animal risible, sans preiudice de son vsage de la raison, recognoissans que cette qualité n'appartient qu’à luy seul. Or puisque le rire est tousiours tesmoin d'vne certaine resiouïssance que nous sentons interieurement, qui de sa nature nous attire au plaisir, $\&$ nous fait desirer la recreation; de là vient que plusieurs gaillardises ont esté inuentées par les hommes; \& n'est pas sans cause que les plus beaux Esprits, \& les plus illustres personnages ont par fois donné tréues à leurs plus serieuses occupations pour dechasser l'ennuyeuse melãcolie par quelque entretien ioyeux.

Suit une petite liste de catégories sociales ou professionnelles qui ont choisi le rire pour se distraire de leurs peines ou de leur travail (artisans, laboureurs, prisonniers...). Cette légitimation universelle du rire se voit encore renforcée par la caution d'une parole d'autorité (Sénèque), sous la forme d'énoncés maximaires. Mais la recherche d'une position médiane impose un effort de relativisation; entre les mariniers et les condamnés à mort, Garon mentionne l'exemple des hommes de foi, hardiesse qu'un quasioxymore peut seul autoriser: "les plus deuots Religieux ne refusent pas de se recreer hõnestement». Il faut, pour rester décent, se modérer dans ses transports, et le rire, pour échapper à toute suspicion, ne peut correspondre qu'à une "douce allégresse». Cette modération sera la preuve que le but moral aura été atteint, car la poétique du rire mise en place par Garon est davantage une poétique du docere qu'une poétique du placere. On recherche les preuves les plus assurées que «la ioye nous est tres-vtile». L'extrême fin de ce texte liminaire insiste sur l'idée de retenue, et comme l'auteur conclut de façon quelque peu cavalière, c'est bien ce dernier conseil qui prévaut:

Ie ne veus pas dire pourtant que la ioye ne doiue estre moderée, \& que nous ne deuions la temperer au milieu de nos plus grandes prosperitez, ne faisans cóme Polycrite, qui ayant fait recouurer la ville de Milet à ses Citoyens, mourut de ioye à la porte. Les mal-aduisez se desbordent en leurs douleurs, außi bien qu'en leurs ioyes. Ie fournirois plusieurs authoritez sur ce sujet, mais pour n'ennuyer le Lecteur, venons à nostre Chasse-ennuy.

Venons-en donc au Chasse-ennuy, puisque l'auteur nous y invite...

Chaque petit récit - qui excède rarement une page entière - est accompagné d'un titre, qui peut simplement signaler le thème traité («L'estime que

I4. Voir l'ouvrage désormais classique de G. Genette, Seuils, Paris, Seuil (Poétique) ou réédition Points Essais, 1987, en particulier le chapitre «Fonctions de la préface originale». 
Louys XII. faisoit des Venitiens», "D'un qui cherchoit sa femme qui s'estoit noyee»), mais aussi orienter la lecture: lecture amusée et admirative dans le cas d'une "belle repartie», d'une "response subtile» ou d'un "dict remarquable \& picquant», lecture railleuse très souvent ("response d'un Duc de Mantouë à un importun", "Belle response qu'il fit à vn vanteur »...).

Si le titre ne suffit pas à distribuer les rôles, c'est souvent le début du récit qui s'en charge. Syntaxiquement, cela se traduit par une abondance très remarquable des expansions du nom (adjectifs, relatives explicatives ou groupes participiaux...). Il est d'ailleurs préférable de se fier à ces indices plutôt qu'à une appartenance sociale, géographique ou professionnelle particulière, car Garon aime beaucoup jouer de l'inversion des stéréotypes: le Dauphinois ou le paysan ne sont pas toujours les brutes supposées, et il arrive aux maîtres en rhétorique (juges, évêques ou écoliers) de se faire surprendre:

LXVI.

Subtile repartie d'vn Paysan Dauphinois à des Escoliers de Lyon.

Vne troupe d'Escoliers estudians à Lyon, allans vn Ieudy au pourmenoir du costé de la Guillotiere, rencontrerent vn Paysan monté sur son Asne, qui se mit à braire au mesme instant qu'il fut proche d'eux. Ceux-cy voulans gausser le Villageois, luy dirent; Ne sçais-tu pas mieux instruire ta beste qui brait hors de saison? Il leur respondit, Mon Asne, Messieurs, est si spirituel \& bien appris, que non seulement, comme font les autres, il chante au mois de May, mais toutes les fois qu'il rencontre quelque brigade de ses freres, en signe de grande liesse il se met à rossignoler, comme vous l'auez oüy maintenãt. À cette response nos Escoliers demeurerent auec moins d'vn pied \& demy de nez.15

Lorsque le lecteur a toutes les cartes en main, le récit s'accélère brutalement, et laisse place, en régime de discours direct, à la parole vive des personnages. Il s'agit d'une technique simple, mais parfaitement éprouvée, sans doute révélatrice d'habitudes liées au genre plus que d'un véritable style d'auteur. Pour ce qui est de l'art de la mise en scène, Garon se contente de mettre en ouvre - souvent avec talent - des recettes déjà maintes fois éprouvées.

Plus caractéristique de sa manière propre est son attachement à la vérité historique. Nous avons signalé plus haut (note 2) un exemple significatif de datation. Cette pratique est loin d'être isolée, et nombreux sont les repères temporels qui permettent de situer, avec plus ou moins de précision, telle ou telle anecdote. Garon s'installe parfois lui-même, quand il le peut, en posture de témoin. Ceci nous vaut quelques spectaculaires irruptions de la première personne:

15. Le Chasse-ennuy, op. cit., p. 274-275. 
Ce que recognoissant Monsieur le Magister, porté de ie ne sçay quel desdain, dit en presence de quelques honnestes personnes, voire à moy en particulier: Ce lourdaut, cette grosse teste, il y a trois ans que ie me peine à l'enseigner $[\ldots]^{16}$

En cela, le texte relève bien du genre de l'anecdote, par essence assez flou, mais auquel Pierre Glaudes a contribué à donner des contours définitoires relativement stables grâce à une précieuse mise au point terminologique:

Si l'on considère, d'autre part, sa valeur de vérité, l'anecdote au même titre que la légende, est par essence testimoniale. Elle tend toujours à s'authentifier par des procédés variés, permettant, selon les cas, de la présenter comme la relation d'un témoignage direct, de choses lues chez des auteurs faisant autorité ou simplement d'ouï-dire plus ou moins sûrs. À cet égard, tout récit anecdotique reste peu ou prou dans les limites du vraisemblable et se présente comme une histoire vérifiable, ce qui n'empêche pas la véracité du témoignage proposé d'être souvent sujette à caution. ${ }^{17}$

Ce souci de vérité qui pousse ici l'auteur à se mettre lui-même en scène, l'amène parfois à parodier la parlure de tel ou tel personnage célèbre. Ainsi Louis XI, sous la plume de Garon, ne peut-il lâcher une phrase sans l'entrecouper d'un juron caractéristique: «Pasque Dieu!» "Tel estoit son juron», glose l'auteur, à l'intention des lecteurs inattentifs. Curieuse méthode que celle qui consiste à multiplier les faux "effets de réel» tout en signalant l'imposture... ${ }^{\text {I } 8}$

De façon générale, que l'on interprète cela comme un défaut ou une qualité, Garon penche pour l'explicitation maximale. Alors que, selon Pierre Glaudes, la leçon de l'anecdote doit rester sous-jacente ${ }^{19}$, notre auteur n'hésite pas à prêter main-forte au lecteur dans son travail de déduction ou d'interprétation, en insérant des maximes parfaitement explicites:

Il vouloit, comme ie croy, inferer de là ; Qu'il n'y a pas plus de peine à acquerir des richesses, qu'il y a de trauaux à les posseder.

Le Roy pour vne si prompte $\&$ gracieuse response, ayant auparauant deliberé de le faire pendre, luy pardonna, pour faire paroistre, qu'entre les Princes benins la iustice cede à la misericorde, \& que le Roy est contraire du Tyran. ${ }^{20}$

I6. Ibid., seconde centurie, LX, p. 270. Nous soulignons.

I7. P. Glaudes, «"Ceci n'est pas même une anecdote”, Approche pragmatique et typologique de l'anecdote, à propos de L'Abyssinien de L. Bloy", dans L'Anecdote (sous la dir. de A. Montandon), Clermont-Ferrand, faculté des Lettres et Sciences humaines de l'université BlaisePascal, fasc. 3I, p. 25

I8. On pourrait multiplier les exemples, avec le fameux «Ventre Saint-Gris!» du bon roi Henri IV, ou plus subtilement, avec des exemples de parodie du langage juridique, attestant chez notre auteur d'une connaissance parfaite du prétoire et de son sociolecte.

19. Ibid., p. 32.

20. Le Chasse-ennuy, op. cit., première centurie, V et XCII, p. 7-8 et 92. 
On voit à de pareils exemples que le rire, institué par les seuils du recueil comme la clé de lecture primordiale, reste parfaitement subordonné aux exigences du docere. Mais cela était, d'une certaine façon, annoncé. Ce qui est plus surprenant est la disparition, à intervalles réguliers, de toute esthétique du rire, au profit de la moralité: la troisième centurie comporte même une sous-section intitulée "moralitez" (\$ LXXXIX sq.), où l'on s'émerveille de la sage législation des Égyptiens ou des Lacédémoniens. Un dernier glissement peut se produire, enfin, lorsque la moralité elle-même disparait de l'horizon de lecture. Les mœurs des Turcs semblent tellement étranges à l'auteur, qu'il consacre une notice à la condition des femmes:

\section{$\mathrm{XX}$.}

Les Turcs sur toutes nations sont ialoux de leurs femmes.

S’il y a des gens au monde soigneux de la pudicité de leurs femmes, ce sont les Turcs: pourtant les tiennent-ils encloses, \& tellement cachees en leurs maisons, qu’à peine le Soleil les void-il. Si la necessité les tire en public, ils les enuoyent tellement couuertes, voilees \& enveloppées, qu'on diroit à leur rencontre que ce sont fantosmes, ou mascarades. Elles voyent les hommes à trauers vn voile, ou resuel: mais nul homme ne void aucune Partie de leur corps. Car tous ont ceste opinion qu'vne femme de beau visage, ou en fleur d'aage ne peut estre veuë d'homme sans conuoitise d'en iouyr, par consequent sans souilleure de cœu: à raison dequoy ils les tiennent toutes cachees.

Le rire ou la raillerie, ici, sont totalement absents. De moralité, il n'est guère question non plus: ni condamnation, ni louange apparentes. Ici, l'esthétique de l'ouvrage s'infléchit, et le Chasse-ennuy devient une sorte de cabinet de curiosités, où l'on enregistre simplement les comportements surprenants, sans prendre parti.

Ainsi, le texte se présente comme une vaste énigme, en ce qu'il multiplie les contradictions internes sans souci apparent de cohérence, et parfois même accentue ces tensions au lieu de les dissimuler: tension entre plusieurs formes de rire parfois peu compatibles, du rire policé au rire grivois; tension entre le rire et l'exigence de moralité, deux visées parfois complémentaires, mais parfois difficilement conciliables; tension entre la raillerie, le rire engagé et l'observation la plus neutre; tension enfin entre une culture humaniste omniprésente, et les aspirations plus modernes du premier baroque: autant de paradoxes qui relèvent le goût de cette salade insolite, que je voulais offrir ici à un critique avisé, et à un gastronome de renom. 